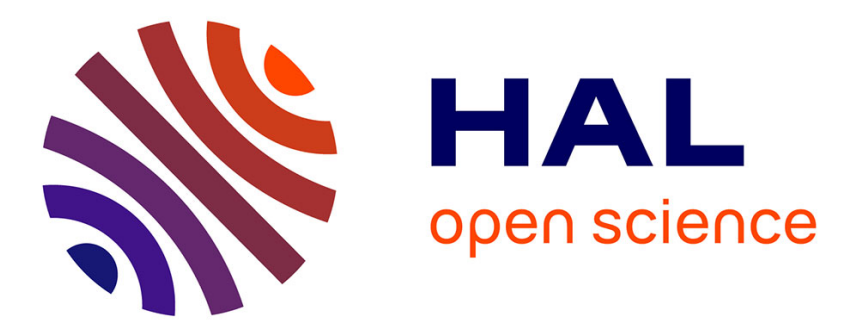

\title{
Optimal Experiment Design for the Identification of One Module in the Interconnection of Locally Controlled Systems
}

Federico Morelli, Xavier Bombois, H. Hjalmarsson, Laurent Bako, Kévin Colin

\section{- To cite this version:}

Federico Morelli, Xavier Bombois, H. Hjalmarsson, Laurent Bako, Kévin Colin. Optimal Experiment Design for the Identification of One Module in the Interconnection of Locally Controlled Systems. 2019. hal-02063406

\author{
HAL Id: hal-02063406 \\ https://hal.science/hal-02063406 \\ Preprint submitted on 11 Mar 2019
}

HAL is a multi-disciplinary open access archive for the deposit and dissemination of scientific research documents, whether they are published or not. The documents may come from teaching and research institutions in France or abroad, or from public or private research centers.
L'archive ouverte pluridisciplinaire HAL, est destinée au dépôt et à la diffusion de documents scientifiques de niveau recherche, publiés ou non, émanant des établissements d'enseignement et de recherche français ou étrangers, des laboratoires publics ou privés. 


\title{
Optimal Experiment Design for the Identification of One Module in the Interconnection of Locally Controlled Systems
}

\author{
F. Morelli ${ }^{1}$, X. Bombois ${ }^{1}$, H.Hjalmarsson ${ }^{2}$, L. Bako ${ }^{1}$ and K. Colin ${ }^{1}$
}

\begin{abstract}
In this paper, we consider the problem of designing the least costly experiment that leads to a sufficiently accurate estimate of one module in a network of locally controlled systems. A module in such a network can be identified by exciting the corresponding local closed loop system. Such an excitation signal will not only perturb the input/output of the to-be-identified module, but also other modules due to the interconnection. Consequently, the cost of the identification can be expressed as the sum of the influence of the excitation signal on the inputs and outputs of all locally controlled systems. We develop a methodology to design the spectrum of the excitation signal in such a way that this cost is minimized while guaranteeing a certain accuracy for the identified model. We also propose an alternative identification configuration which can further reduce the propagation of the excitation signal to other modules and we make steps to robustify this optimal experiment design problem with respect to the cost of the identification.
\end{abstract}

\section{INTRODUCTION}

Nowadays, applications of dynamic networks are more and more widespread. The interconnection of locally controlled systems is a particular type of networked system: each system/agent has its own control strategy, while at the same time it shares information with the other agents in order to reach a global objective [1]. A typical example may be a group of drones following a common target [2]. The interconnection of locally controlled systems can be found in civil applications, e.g. Automated Highway Systems [3], as well as in post-emergency scenario e.g. [4]. In these applications, undesired behaviour of one system affects the behaviour of the others, thus possibly degrading the global behaviour of the network. This situation is particularly critical when these systems operate in a dangerous environment, as in a post-emergency scenario [4]. In these situations, when we notice that the performance of one local controller degrades, we need to redesign it using an appropriate model, obtained while the network is operating.

System Identification provides techniques for obtaining a model for a system directly from experimental data [5], thus providing a suitable model for designing a controller [6]. An external excitation signal is added to the input of the system for a fixed time, then the input-output data are collected and the model is obtained by means of an identification criterion, as in Prediction Error Methods. These methods have been extended only recently to networked systems [7], providing conditions for obtaining a consistent estimate of only one module inside the network [8]. Under certain conditions we can define an uncertainty region associated to the model, containing the true system [5], which can be used to design a controller that robustly ensures the network performance [9]. This uncertainty region can be shaped by a proper design of the external excitation signal used during the experiment.

In all the situations where we cannot stop all the systems in the network, performing an identification experiment will alter the normal behaviour of the system, thus affecting the whole network. In order to reduce the effect of the excitation signal on the normal behavior of the network, we extend in this paper the concept of least costly experiment design introduced in [6]. More precisely, we consider the case where one single module of the network has to be identified and we determine the excitation signal for this identification which induces the least perturbation on the global behaviour of the network, while guaranteeing a certain level of accuracy for the identified model. The induced perturbation (i.e. the cost of the identification) is defined as the sum of the influence of the excitation signal on the input and the output of each module.

Building upon the concept of stealth identification that we introduced in [10], we also propose a new identification configuration that will further reduce the cost due to the propagation of the excitation signal applied to the to-be-identified module towards the other modules. This is achieved via an initial model of the to-be-identified system that is used to remove an estimate of the contribution of the excitation signal from the signal that will be propagated to the other modules. This is a new application of the stealth identification concept since, in [10], it was introduced as a tool to enable classical optimal experiment design in a loop where the controller is not Linear Time Invariant (LTI). Moreover, another contribution of the present paper is to robustify the stealth approach by considering the uncertainty of this initial model and its influence on the cost of the identification.

Notations: $\mathbb{C}$ is the set of complex numbers. For $z \in \mathbb{C},|z|$ is the modulus of $z$. For $d_{1}, d_{2}, \ldots, d_{n} \in \mathbb{R}, D=\operatorname{diag}\left\{d_{1}, d_{2}, \ldots, d_{n}\right\}$ denotes the diagonal matrix $D \in \mathbb{R}^{n \times n}$ with the elements $d_{1}, d_{2}, \ldots, d_{n}$ on its main diagonal.

\footnotetext{
${ }^{1}$ Laboratoire Ampère, Ecole Centrale de Lyon, 36 avenue Guy de Collongue, 69134 Ecully Cedex, France federico.morelli@ec-lyon.fr

2 Automatic Control, School of Electrical Engineering, KTH, 10044 Stockholm, Sweden
} 


\section{SYSTEM IDENTIFICATION FOR LOCALLY CONTROLLED SYSTEM}

\section{A. Interconnection of Locally Controlled Systems}

Let us consider a network made of $N_{m}$ modules, where each module $\mathscr{N}_{i}$ contains a Linear Time Invariant (LTI) system $\mathscr{S}_{i}$, described by a Single Input, Single Output (SISO) discrete-time stable transfer function $G_{0, i}(z)$. Its output, $y_{i}(t)$, is affected by a disturbance $v_{i}(t)=H_{0, i}(z) e_{i}(t)$, where $e_{i}(t)$ is a white noise of variance $\sigma_{i}^{2}$ and $H_{0, i}(z)$ is a stable linear transfer function, assumed to be monic and minimum-phase. The system is operated in closed loop by means of a local LTI controller $K_{i}(z)$ such that the output follows a local reference $y_{r e f, i}(t)$, as in Fig. 1 . We can describe a generic $\mathscr{N}_{i}, i=1, \ldots, N_{m}$, as:

$$
\left\{\begin{array}{l}
y_{i}(t)=G_{0, i}(z) u_{i}(t)+v_{i}(t) \\
u_{i}(t)=K_{i}(z)\left(y_{\text {ref }, i}(t)-y_{i}(t)\right)
\end{array}\right.
$$

Each module $\mathscr{N}_{i}$ is made of the system $\mathscr{S}_{i}$ and its closed-loop controller $K_{i}(z)$, as in Fig. 1. The true system of each module is denoted by $\mathscr{S}_{i}=\left\{G_{0, i}, H_{0, i}\right\}$, while the modules are denoted by $\mathscr{N}_{i}=\left\{K_{i}, \mathscr{S}_{i}\right\}$.

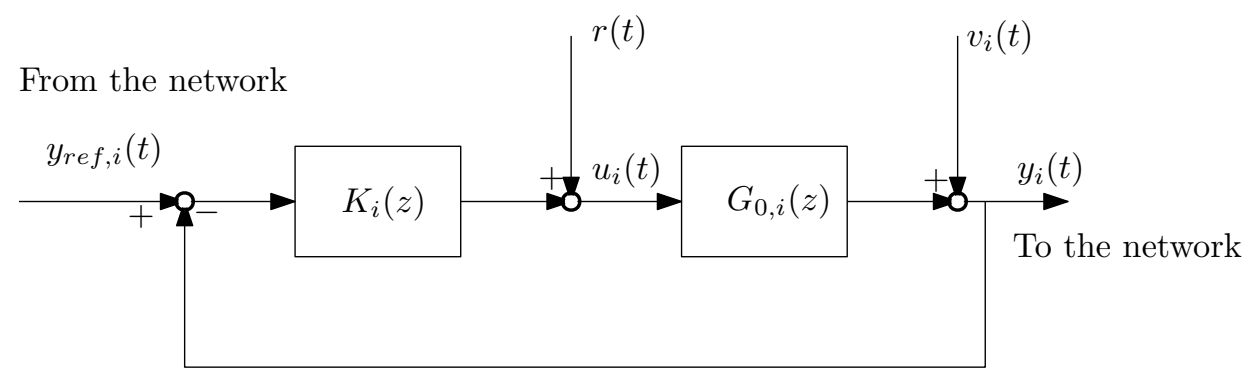

Fig. 1. Representation of a single module $\mathscr{N}_{i}$

While we can describe each module independently using (1), the local reference $y_{r e f, i}(t)$ depends on the output signals of the other modules inside the network [2]. This dependence can be described using a graph representation, see e.g. Fig. 2, where each node represents a module and the edges represent the communication links between them. A consensus strategy [1] determines how the information, coming from the nodes, is used for computing $y_{r e f, i}(t)$.

Let us consider, as an illustrative example, the network depicted in Fig. 2, where $y_{r e f, i}(t), i=1, \ldots, N_{m}$, is computed as the average of the entering output signals, e.g. $y_{r e f, 3}(t)=\frac{y_{2}(t)+y_{5}(t)}{2}$ in Fig. 2. Moreover, each output $y_{i}(t)$ must follow a global reference $r e f_{\text {ext }}(t)$, given to only certain nodes in the network. Let $A \in \mathbb{R}^{N_{m} \times N_{m}}$ be the adjacency matrix associated to the graph, defined such that its $i, j^{\text {th }}$ entry is 1 if there exists a directed edge from the node $j$ to the node $i$. Let $d(i)$ be the in-degree of the $i^{t h}$ node, defined as the number of directed edges entering the node $i$, and $D \in \mathbb{R}^{N_{m} \times N_{m}}$ the in-degree matrix, defined as $D=\operatorname{diag}\left\{d(1), d(2), \ldots, d\left(N_{m}\right)\right\}$. By grouping the signals $y_{r e f, i}(t)$ in a column vector $\bar{y}_{\text {ref }}(t)=\left(y_{\text {ref }, 1}(t), y_{\text {ref }, 2}(t), \ldots, y_{\text {ref }, N_{m}}(t)\right)^{T}$ and the output signals in a column vector $\bar{y}(t)=\left(y_{1}(t), y_{2}(t), \ldots, y_{N_{m}}(t)\right)^{T}$, we can write this consensus strategy as:

$$
\bar{y}_{\text {ref }}(t)=A_{d} \bar{y}(t)+B r e f_{\text {ext }}(t)
$$

where $A_{d}$ is the normalized adjacency matrix, defined as $A_{d}=D^{-1} A$, and $B$ is the input matrix for the global external reference signal $r e f_{e x t}(t)\left(B=(1,0, \ldots, 0)^{T}\right.$ for the network in Fig. 2). Despite the simplicity of this example, a general consensus strategy can always be written in this form [2]. It is important to note that each module $\mathscr{N}_{i}$ is independently described by (1), but the presence of a term obtained through a consensus strategy (2) couples the behaviour of the modules [2]. Then the input signal $u_{i}(t)$ of $\mathscr{N}_{i}$ depends also on the output $y_{k}(t)$ of each module $\mathscr{N}_{k}$ having a path to $\mathscr{N}_{i}$.

\section{B. Identification of One Module}

In this paper, we will suppose that we need to (re-)identify one of the $N_{m}$ modules of the network. In the sequel, the index of this particular module will be denoted by $l \in\left\{1,2, \ldots, N_{m}\right\}$ and the remaining modules will be denoted by $k \in\left\{1,2, \ldots, N_{m}\right\} \backslash l$. The index $i \in\left\{1,2, \ldots, N_{m}\right\}$ will be used when we refer to every module in general. For identification purpose, we excite the module $l$ using a (sufficiently exciting) excitation signal $r(t)$, then (1) for $u_{l}(t)$ in $\mathscr{N}_{l}$ is modified to:

$$
u_{l}(t)=r(t)+K_{l}(z)\left(y_{r e f, l}(t)-y_{l}(t)\right)
$$

while $u_{k}(t)$ remains as in (1) for $k \neq l$.

As shown in [9], we can use Prediction Error (PE) identification to identify the system $\mathscr{S}_{l}$ in the network by using a set of input-output experimental data $Z^{N}=\left\{u_{l}(t), y_{l}(t) \quad t=1, \ldots, N\right\}$. Indeed, using a full-order parametric model structure 


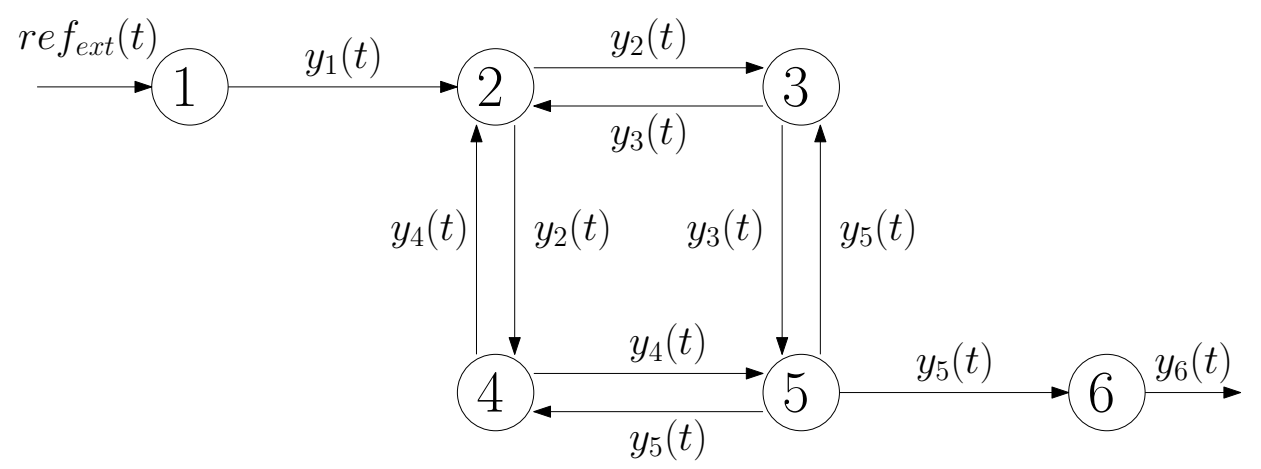

Fig. 2. Example of graph representation of the network, each node represents a module $\mathscr{N}_{i}$ and the edges represent the communication link between the modules

$\mathscr{M}=\left\{G\left(z, \theta_{l}\right), H\left(z, \theta_{l}\right)\right\}, \theta_{l} \in \mathbb{R}^{n}$, we can deduce a consistent estimate $\hat{\theta}_{N, l}$ of $\theta_{0, l}$, named true parameter vector ${ }^{1}$, using the following PE criterion:

$$
\begin{aligned}
\hat{\theta}_{N, l} & =\underset{\theta_{l}}{\arg \min } \frac{1}{N} \sum_{t=1}^{N} \varepsilon^{2}\left(t, \theta_{l}\right) \\
\varepsilon\left(t, \theta_{l}\right) & =H^{-1}\left(z, \theta_{l}\right)\left(y_{l}(t)-G\left(z, \theta_{l}\right) u_{l}(t)\right)
\end{aligned}
$$

Moreover, this consistent estimate is asymptotically normally distributed around $\theta_{0, l}$, with asymptotic covariance $P_{\theta}$, [5]. It is shown in [9] that $P_{\theta}^{-1}$ can be written as an affine function of $\Phi_{r}(\omega)$, the power spectrum of the excitation $r(t)$. This function is dependent on $\theta_{0}$, made up of the concatenation of the true parameter vectors $\theta_{0, i}$ of all modules $\left(i=1, \ldots, N_{m}\right)$. Consequently we denote the inverse of the covariance matrix as $P_{\theta}^{-1}\left(\Phi_{r}, \theta_{0}\right)$.

Remark 1: For simplicity we will suppose that $r e f_{\text {ext }}(t)=0$ only during the identification experiment [9].

\section{OPTIMAL EXPERIMENT DESIGN}

In this paper, we consider the following optimal experiment design problem:

Problem 1 (Optimal experiment design):

For an identification of duration $N$, described in Section II-B, determine the power spectrum $\Phi_{r}$ of the excitation signal $r(t)$ leading to the smallest identification cost, while guaranteeing that the identified parameter vector is sufficiently accurate.

The accuracy requirement will be of the form $P_{\theta}^{-1}\left(\Phi_{r}, \theta_{0}\right)>R_{a d m}$ [11]. As far as the identification cost is concerned, we will extend the notion of cost introduced in [6] to the network case. For this purpose, it is important to note that the excitation signal $r(t)$ will not only influence the module $\mathscr{N}_{l}$, but will also influence the other modules $\mathscr{N}_{k}$, via (2). Therefore we define the identification cost as:

$$
J\left(\Phi_{r}, \theta_{0}\right)=\sum_{i=1}^{N_{m}}\left(\mathscr{P}_{y_{r, i}}\left(\Phi_{r}, \theta_{0}\right)+\eta_{u} \mathscr{P}_{u_{r, i}}\left(\Phi_{r}, \theta_{0}\right)\right)
$$

where $y_{r, i}$ and $u_{r, i}$ are the parts of $y_{i}$ and $u_{i}, i \in\left\{1, \ldots, N_{m}\right\}$, induced by the excitation signal $r(t)$ of spectrum $\Phi_{r}(\omega)$ and $\eta_{u}$ is an user chosen scalar. Finally, given a signal $h(t)$, which is a filtered version of $r(t)$ via a transfer function $F(z, \theta)$ parametrized by a vector $\theta \in \mathbb{R}^{n}$ i.e. $h(t)=F(z, \theta) r(t)$, the notation $\mathscr{P}_{h}\left(\Phi_{r}, \theta\right)$ denotes the power of $h(t)$ i.e. $\frac{1}{2 \pi} \int_{-\pi}^{\pi}\left|F\left(e^{j \omega}, \theta\right)\right|^{2} \Phi_{r}(\omega) d \omega$. The transfer function between $r(t)$ and the signals $y_{r, i}$ (resp $u_{r, i}$ ) may depend on all the systems $\mathscr{S}_{i}$ and thus on the whole vector $\theta_{0}$. Note that, in (5), the elements $\mathscr{P}_{y_{r, i}}$ and $\mathscr{P}_{u_{r, i}}$, corresponding to a module $\mathscr{N}_{i}$ to whom $\mathscr{N}_{l}$ does not have a path will be identically zero.

As in [6], the cost $J\left(\Phi_{r}, \theta_{0}\right)$ is affine in the decision variable $\Phi_{r}$ and we already mentioned that this is also the case for $P_{\theta}^{-1}\left(\Phi_{r}, \theta_{0}\right)$. Consequently, if we replace $\theta_{0}$ by an initial estimate, we can derive the optimal power spectrum using convex optimization.

However, replacing the true $\theta_{0}$ by an initial estimate has two important consequences: the obtained accuracy could be lower than the expected one and the entailed cost of the experiment could be higher. As pointed out in [12], the second consequence may be the most critical in practice. Consequently, in this paper, we will try to reduce as much as possible the risk of this second consequence by robustifying the cost $J$ of our optimal experiment design problem with respect to the uncertainty of the initial estimate. It is important to note that $\mathscr{P}_{y_{r, l}}\left(\right.$ resp $\mathscr{P}_{u_{r, l}}$ ) will be much larger than $\mathscr{P}_{y_{r, k}}\left(\right.$ resp $\mathscr{P}_{u_{r, k}}$ ) due to the network attenuation. Moreover, $\mathscr{P}_{y_{r, l}}$ and $\mathscr{P}_{u_{r, l}}$ will be mainly influenced by the dynamic of the $l^{\text {th }}$ module. Consequently, even though we could extend our results to take into account the uncertainties of all initial estimates, we will

$$
{ }^{1} \theta_{0, l} \text { is s.t. } G\left(z, \theta_{0, l}\right)=G_{0, l}(z) \text { and } H\left(z, \theta_{0, l}\right)=H_{0, l}(z)[5]
$$


only consider the uncertainty of the initial estimate $\theta_{\text {init }, l}$ of $\theta_{0, l}$ to robustify the cost. This means that we will still replace the true parameter vectors $\theta_{0, k}, k \neq l$, in (5) by their initial estimates $\theta_{\text {init }, k}$. By doing this, $J$ and the functions involved in its definition become functions of only $\theta_{0, l}$ and $\Phi_{r}$, so we can rewrite (5):

$$
J\left(\Phi_{r}, \theta_{0, l}\right)=\sum_{i=1}^{N_{m}}\left(\mathscr{P}_{y_{r, i}}\left(\Phi_{r}, \theta_{0, l}\right)+\eta_{u} \mathscr{P}_{u_{r, i}}\left(\Phi_{r}, \theta_{0, l}\right)\right)
$$

To define the uncertainty of $\theta_{\text {init.l }}$, we suppose that this initial estimate has been determined via an initial identification experiment with covariance matrix $P_{i n i t}$. Consequently, we know that $\theta_{0, l}$ lies, with an user-chosen probability level $\beta$, in the following ellipsoidal region $U_{\text {init }}$ :

$$
U_{\text {init }}:=\left\{\theta_{l} \mid\left(\theta_{l}-\theta_{\text {init }, l}\right)^{T} P_{\text {init }}^{-1}\left(\theta_{l}-\theta_{\text {init }, l}\right) \leq \chi_{\beta}\right\}
$$

where $\chi_{\beta}$ is s.t. $\operatorname{Pr}\left(\chi^{2}(n) \leq \beta\right)=\chi_{\beta}$.

Finally, we can reformulate the optimal experiment design problem as follows:

Problem 2 (Robust optimal experiment design):

Determine the power spectrum $\Phi_{r}(\omega)$ of the signal $r(t)$ which solves the following minimization problem:

$$
\begin{gathered}
\min _{\Phi_{r}, \gamma} \gamma \\
J\left(\Phi_{r}, \theta_{l}\right) \leq \gamma \quad \forall \theta_{l} \in U_{\text {init }} \\
P_{\theta}^{-1}\left(\Phi_{r}, \theta_{\text {init }}\right) \geq R_{\text {adm }}
\end{gathered}
$$

where $J\left(\theta_{l}, \Phi_{r}\right)$ is defined in (6) and $\theta_{\text {init }}$ is the initial estimate of the whole parameter vector $\theta_{0}$.

Observe that, as already mentioned, only the cost $J$ is robustified, since for the accuracy constraint in (9) we have replaced $\theta_{0}$ by its initial estimate $\theta_{\text {init }}$. Indeed, we need to robustify the cost in order to prevent undesired behaviour of the network, but, if the new model does not respect the accuracy requirement, it is sufficient to perform another identification experiment.

Remark 2: Note that the matrix $R_{a d m}$ can be defined as $R_{a d m, d e s}-P_{\text {init }}^{-1}$, where $R_{a d m, \text { des }}$ represents the desired accuracy and $P_{\text {init }}$ is the covariance matrix of the initial estimate. In this case, the estimate obtained from the to-be-designed experiment will be combined with the initial estimate $\theta_{\text {init, }, l}$, leading to the final estimate of $\theta_{0, l}$ (see e.g. [13]).

Remark 3: The existing tools in Robust Experiment Design literature are based on Taylor approximations [13], [12], or on a Monte-Carlo like approach [14], [6], thus not completely ensuring that the actual cost will be below the minimized one. The solution presented above, considering only the uncertainty of the to-be-identified module, is obviously fully exact when there is only one module in the network i.e. when we are in the classical closed-loop identification setting. In Section IV we will present a procedure that will allow to solve Problem 2 by replacing (8) by a convex constraint implying (8). Consequently, if we use this procedure in the single closed-loop case, it provides us a tool that, unlike the methods involving approximations, will guarantee us that the cost will remain below a certain threshold.

Besides proposing a method to solve the above problem using convex optimization, we also propose an identification experiment configuration which allows to reduce even further the cost related to the modules $\mathscr{N}_{k}$ i.e. to reduce the propagation of the effects of $r(t)$ through the network. For this purpose, we use the concept, introduced in [10], of Stealth Identification. In [10], we used this configuration in order to enable classical optimal experiment design in a closed-loop configuration when the controller is not LTI. Here it will be used as a tool to reduce the propagation of the excitation signal to the other modules.

Now we present how to use the concept of Stealth Identification for this purpose. The closed-loop in the to-be-identified module $\mathscr{N}_{l}$ is modified as follows:

$$
\begin{aligned}
y_{l}(t) & =G_{0, l}(z) u_{l}(t)+v_{l}(t) \\
u_{l}(t) & =r(t)+K_{l}(z)\left(y_{\text {ref }, l}(t)-x(t)\right) \\
x(t) & =y_{l}(t)-G_{\text {init }, l}(z) r(t)
\end{aligned}
$$

where $G_{\text {init }, l}(z)=G_{l}\left(z, \theta_{\text {init }, l}\right)$, with $\theta_{\text {init }, l}$ the initial estimate of $\theta_{0, l}$. The modified loop is represented in Fig 3, where $r(t)$ is the to-be-designed excitation signal. As before, we collect $N$ input-output data points in $Z^{N}=\left\{y_{l}(t), u_{l}(t) \quad t=1, \ldots, N\right\}$ in order to identify $\hat{\theta}_{N, l}$ using (4). The signal $x(t)$, defined in (11) as the difference between the output signal $y_{l}(t)$ and $G_{\text {init }, l}(z) r(t)$, is used by the controller $K_{l}$ instead of $y_{l}(t)$. Moreover, $x(t)$ will be used to compute $\bar{y}_{\text {ref }}(t)$ as well:

$$
\bar{y}_{r e f}(t)=A_{d} \bar{y}_{x}(t)
$$

where $\bar{y}_{x}^{T}(t)=\left(y_{1}(t), \ldots, y_{l-1}(t), x(t), y_{l+1}(t), \ldots, y_{N_{m}}(t)\right)$. Let us recall that the signal $r e f_{\text {ext }}(t)=0$ during the identification experiment.

In order to explain this new configuration, let us first consider the ideal case where $G_{\text {init }, l}=G_{0, l}$. In this ideal case, the signal $x(t)$ is independent of the excitation $r(t)$ (i.e. the transfer function between $r(t)$ and $x(t)$ is identically zero). Since $x(t)$ is used in (12) to compute $\bar{y}_{r e f}(t)$, the excitation $r(t)$ will therefore have no influence on $y_{k}(t)(k \neq l)$ and $u_{k}(t)(k \neq l)$. 


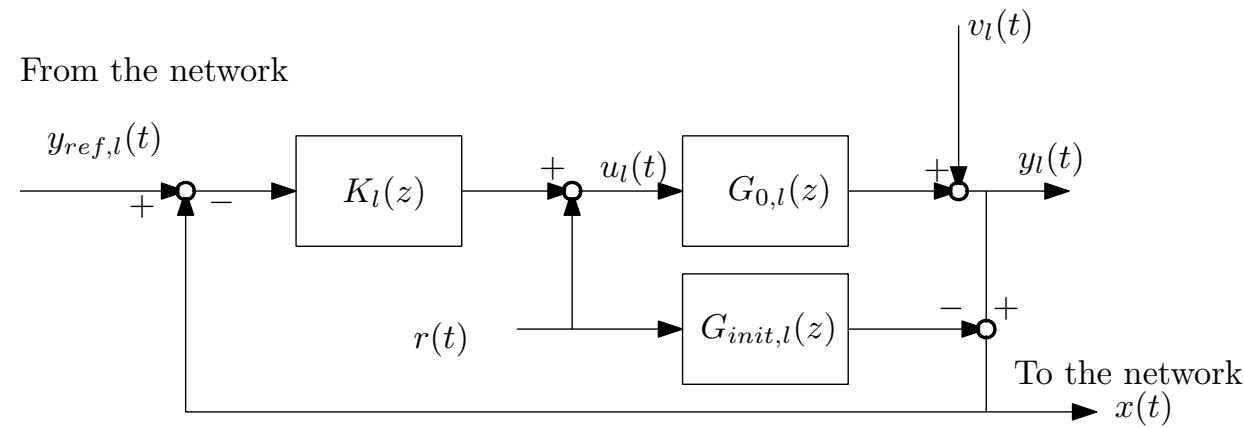

Fig. 3. Stealth Configuration in the module $\mathscr{N}_{l}$

In the non-ideal case, assuming $U_{\text {init }}$ to be small enough, the transfer function between $r(t)$ and $x(t)$ is of course nonzero, but the influence of $r(t)$ on $x(t)$ will generally be smaller than the influence on $y_{l}(t)$ in the original configuration. This is specially the case when the power spectrum $\Phi_{r}$ is designed to reduce the cost $J\left(\Phi_{r}, \theta_{0}\right)$. Consequently, it makes sense to consider the robust optimal experiment design problem, but to apply it when the network follows this new configuration. From a mathematical point-of-view, both problems are equivalent (for what concern the complexity), but it is expected that the optimal cost will be smaller when using the stealth configuration (at least if the uncertainty $U_{\text {init }}$ is small enough).

Remark 4: We have already mentioned the novel use of the Stealth configuration as a difference with our earlier contribution [10]. Another important difference is also the fact that the spectrum of the excitation signal will be optimized while taking into account the uncertainty of $G_{i n i t, l}$. In [10], this spectrum was designed under the assumption $G_{i n i t, l}=G_{0, l}$.

\section{ROBUSTIFICATION OF THE COST}

In order to convexify the robust optimal experiment design problem, presented in the previous section, we only have to replace the constraint (8) by a convex one, ensuring that (8) holds. To this aim, we restrict our attention to signals $r(t)$ given by the summation of a finite number of $n_{\omega}$ sine signals, with known frequencies $\omega_{m}$ and phases $\phi_{m}$, but unknown amplitudes $A_{m}, m=1, \ldots, n_{\omega}$. Then the signal $r(t)$ and its power spectrum $\Phi_{r}(\omega)$ are given by:

$$
\left\{\begin{array}{l}
r(t)=\sum_{m=1}^{n_{\omega}} A_{m} \sin \left(\omega_{m} t+\phi_{m}\right) \\
\Phi_{r}(\omega)=\sum_{m=1}^{n_{\omega}} \frac{\pi A_{m}^{2}}{2}\left(\delta\left(\omega-\omega_{m}\right)+\delta\left(\omega+\omega_{m}\right)\right)
\end{array}\right.
$$

where $\delta(\omega)$ is the Dirac's delta function.

To develop the convex relaxation mentioned above, let us first rewrite the network configuration by focusing only on the excitation $r(t)$, since the noises do not play any role in (6). Then, recalling our previous assumption that only the uncertainty on $\theta_{\text {init }, l}$ is taken into account, the networked system in the stealth configuration can be described as follows, for $\theta_{l} \in U_{\text {init }}$ :

$$
\begin{aligned}
y_{r, l}(t) & =G\left(z, \theta_{l}\right) u_{r, l}(t) \\
y_{r, k}(t) & =G_{i n i t, k}(z) u_{r, k}(t) \quad \forall k \neq l \\
x_{r}(t) & =y_{r, l}(t)-G_{\text {init }, l}(z) r(t) \\
u_{r, l}(t) & =r(t)+K_{l}(z)\left(y_{r e f, r, l}(t)-x_{r}(t)\right) \\
u_{r, k}(t) & =K_{k}(z)\left(y_{r e f, r, k}(t)-y_{r, k}(t)\right) \quad \forall k \neq l \\
\bar{y}_{r e f, r}(t) & =A_{d} \bar{y}_{x, r}(t) \\
\bar{y}_{x, r}^{T}(t) & =\left(y_{r, 1}(t), \ldots, y_{r l-1}(t), x_{r}(t), y_{r, l+1}(t), \ldots, y_{r, N_{m}}(t)\right)
\end{aligned}
$$

where $G_{\text {init }, k}(z)=G_{k}\left(z, \theta_{\text {init }, k}\right)(k \neq l)$ is the initial model for the system $\mathscr{S}_{k}$ and the additional subscript $r$, e.g. in $y_{r, l}(t)$, denotes the part of the original signal, e.g. $y_{l}(t)$, given by the excitation signal $r(t)$. In the non-stealth configuration we just have to pose $x_{r}(t)=y_{r, l}(t)$ in the above equations.

We have seen in [15] that most of the black-box parametrizations used in identification lead to a parametrization $G\left(z, \theta_{l}\right)$ which is an LFT in $\theta_{l}$ [16]. Using the system of equations above, we can rewrite the transfer function between $r(t)$ and any $y_{r, i}(t)$ (resp $\left.u_{r, i}(t)\right)$ as an LFT in $\theta_{l}$. This e.g. means that we can determine $p(t)$ and $q(t)$ such that the transfer function between $r(t)$ and $y_{r, i}(t)\left(\operatorname{resp} u_{r, i}(t)\right)$ can be written as:

$$
\left(\begin{array}{c}
p(t) \\
y_{r, i}(t)
\end{array}\right)=M_{y, i}(z)\left(\begin{array}{c}
q(t) \\
r(t)
\end{array}\right) \quad q(t)=\theta_{l} p(t)
$$

for a given matrix of transfer functions $M_{y, i}(z)\left(\operatorname{resp} M_{u, i}(z)\right)$ that does not depend on $\theta_{l}$. For the LFT representations we will use the notations $y_{r, i}(t)=\mathscr{F}\left(M_{y, i}(z), \theta_{l}\right) r(t)$ and $u_{r, i}(t)=\mathscr{F}\left(M_{u, i}(z), \theta_{l}\right) r(t)$. 
Let us recall also that, given an LFT $\mathscr{F}\left(M_{h}(z), \theta\right)$, we developed in [15] convex tools to exactly compute the quantity $\alpha_{h}^{U}(\omega)$ at a given frequency $\omega$ :

$$
\alpha_{h}^{U}(\omega)=\max _{\theta \in U}\left|\mathscr{F}\left(M_{h}\left(e^{j \omega}\right), \theta\right)\right|^{2}
$$

where $U$ is an ellipsoidal uncertainty region e.g. defined as in (7). We can also deduce the following result:

Proposition 1: Consider a signal $h(t)$, which is a filtered version of $r(t)$, defined as in (13), by means of the transfer function $\mathscr{F}\left(M_{h}(z), \theta\right)$, which is an LFT in a vector $\theta$. Consider an uncertainty region $U$ defined as in (7). Let us define $\mathscr{P}_{h}\left(\Phi_{r}, \theta\right)$ as the power of $h(t)$ for a given $\theta$ :

$$
\mathscr{P}_{h}\left(\Phi_{r}, \theta\right)=\frac{1}{2 \pi} \int_{-\pi}^{\pi}\left|\mathscr{F}\left(M_{h}\left(e^{j \omega}\right), \theta\right)\right|^{2} \Phi_{r}(\omega) d \omega
$$

Then, given $\alpha_{h}^{U}(\omega)$ defined as in (16), the constraint $\mathscr{P}_{h}\left(\Phi_{r}, \theta\right) \leq \gamma \forall \theta \in U$ holds if:

$$
\sum_{m=1}^{n_{\omega}} \frac{A_{m}^{2}}{2} \alpha_{h}^{U}\left(\omega_{m}\right) \leq \gamma
$$

Proof: Given the uncertainty region $U$, defined as in (7), and the signal $r(t)$, defined as in (13), the constraint $\mathscr{P}_{h}\left(\Phi_{r}, \theta\right) \leq \gamma \forall \theta \in U$ holds if and only if the following constraint holds:

$$
\max _{\theta \in U} \sum_{m=1}^{n_{\omega}} \frac{A_{m}^{2}}{2}\left|\mathscr{F}\left(M_{h}\left(e^{j \omega_{m}}\right), \theta\right)\right|^{2} \leq \gamma
$$

Recalling the definition (16), it is immediate to see that:

$$
\max _{\theta \in U} \sum_{m=1}^{n_{\omega}} \frac{A_{m}^{2}}{2}\left|\mathscr{F}\left(M_{h}\left(e^{j \omega_{m}}\right), \theta\right)\right|^{2} \leq \sum_{m=1}^{n_{\omega}} \frac{A_{m}^{2}}{2} \alpha_{h}^{U}\left(\omega_{m}\right)
$$

Consequently, if (18) holds, then (19) holds and thus $\mathscr{P}_{h}\left(\Phi_{r}, \theta\right) \leq \gamma \forall \theta \in U$.

Using Proposition 1, we will be able to replace the constraint (8) in Problem 2 by a constraint similar to (18), which is linear in the square $A_{m}^{2}$ of the to-be-determined amplitudes of the signal $r(t)$. This leads to the following convex optimization problem to tackle the experiment design problem defined in Problem 1.

Problem 3 (Robust optimal experiment design):

Determine the amplitudes $A_{m}^{2}, m=1, \ldots, n_{\omega}$, of the signal $r(t)$, defined as (13), which solve the following minimization problem:

$$
\begin{gathered}
\min _{A_{1}^{2}, \ldots, A_{n_{\omega}}^{2}, \gamma} \gamma \\
\sum_{m=1}^{n_{\omega}} \frac{A_{m}^{2}}{2} \sum_{i=1}^{N_{m}}\left(\alpha_{y_{r, i}}^{U_{\text {init }}}\left(\omega_{m}\right)+\eta_{u} \alpha_{u_{r, i}}^{U_{\text {init }}}\left(\omega_{m}\right)\right) \leq \gamma \\
P_{\theta}^{-1}\left(A_{1}^{2}, \ldots, A_{n_{\omega}}^{2}, \theta_{\text {init }}\right) \geq R_{\text {adm }} \\
A_{m}^{2} \geq 0 \quad m=1, \ldots, n_{\omega}
\end{gathered}
$$

where the terms $\alpha_{y_{r, i}}^{U_{\text {init }}}\left(\right.$ resp $\left.\alpha_{u_{r, i}}^{U_{\text {init }}}\right)$ are computed as in (16), for the signals $y_{r, i}$ (resp $u_{r, i}$ ) and the uncertainty region $U_{\text {init }}$, and where $P_{\theta}^{-1}\left(A_{1}^{2}, \ldots, A_{n_{\omega}}^{2}, \theta_{\text {init }}\right)$ is the affine function in $A_{m}^{2}\left(m=1, \ldots, n_{\omega}\right)$, obtained by replacing $\Phi_{r}$ with (13) in the expression $P_{\theta}^{-1}\left(\Phi_{r}, \theta_{\text {init }}\right)$.

The constraint (21) is also linear in the decision variables $A_{m}^{2}$ and in $\gamma$. Thus Problem 3 is a convex optimization problem, which can be solved efficiently by standard optimization techniques [17].

\section{SIMULATION RESULTS}

Here we report an example in order to illustrate our results. We consider an interconnection of locally controlled systems, whose topology is depicted in Fig. 2 and each module has the same ARX system [18]:

$$
y_{i}(t)=\frac{z^{-3} B_{0}(z)}{A_{0}(z)} u_{i}(t)+\frac{1}{A_{0}(z)} e_{i}(t)
$$

with $A_{0}(z)=1-1.99185 z^{-1}+2.20265 z^{-2}-1.84083 z^{-3}+0.89413 z^{-4}$ and $B_{0}(z)=0.10276-0.18123 z^{-1}$.

All white noises $e_{i}(t)$ have the same variance $\sigma_{i}^{2}=0.5$ and we suppose that every module is actuated using the same controller $K_{i}(z)=\frac{K_{B}(z)}{K_{A}(z)}$ with $K_{B}(z)=0.03742-0.06719 z^{-1}+0.06995 z^{-2}-0.03814 z^{-3}-0.02546 z^{-4}+0.06323 z^{-5}-$ $0.04707 z^{-6}+0.03222 z^{-7}$ and $K_{A}(z)=1-3.348 z^{-1}+5.953 z^{-2}-7.163 z^{-3}+6.143 z^{-4}-3.705 z^{-5}+1.368 z^{-6}-0.2482 z^{-7}$. We consider that it is needed to re-identify the system $\mathscr{S}_{5}$ in the module $\mathscr{N}_{5}$ and that all the modules $\mathscr{N}_{i}$ have the same initial model:

$$
y_{i}(t)=\frac{z^{-3} B_{i, i n i t}(z)}{A_{i, i n i t}(z)} u_{i}(t)+\frac{1}{A_{i, \text { init }}(z)} e_{i}(t)
$$


with $B_{i, \text { init }}(z)=0.08068-0.1671 z^{-1}$ and $A_{i, \text { init }}(z)=1-1.995 z^{-1}+2.187 z^{-2}-1.804 z^{-3}+0.8764 z^{-4}$.

We consider that $R_{a d m, d e s}$ is defined such that the new model must satisfy a requirement on the sensitivity function of the to-be-redesigned controller [6] and that $R_{a d m}$ is computed as in Remark 2. An initial uncertainty region is available, as in (7), with a $\beta=95 \%$ confidence level. We consider a fixed experiment length $N=1000$, the weight $\eta_{u}=1$ and an excitation signal $r(t)$ as in (13), with $n_{\omega}=50$ harmonics taken as equally spaced points in the frequency span $\left[10^{-2}, \pi\right](\mathrm{rad} / \mathrm{s})$.

Stealth Configuration. We use the available initial model to modify $\mathscr{N}_{5}$ as in Fig. 3 (Stealth Configuration). Then we solve Problem 3 using the LMI solver provided in the Robust Control toolbox in Matlab. The solution of the problem provides an optimal power spectrum $\Phi_{r, o p t}$, completely defined by the squared amplitudes $A_{o p t, m}^{2}$, and an optimal cost $\gamma_{o p t}=1.831$.

In order to check the validity of our results, we perform the identification experiment with the designed excitation signal. Then we identify a new model $G_{5}\left(z, \hat{\theta}_{N, 5}\right)$, along with the covariance matrix for $\hat{\theta}_{N, 5}$, and we observe that $\hat{\theta}_{N, 5}$ and its covariance matrix satisfy the accuracy requirement. In order to verify our robustification approach, we decide to compare $\gamma_{o p t}$ with the actual cost $J\left(\Phi_{r, o p t}, \theta_{0}\right)$ computed as in (5) using $\Phi_{r, o p t}$ and $\theta_{0}$, which is here known. We observe that the actual cost $J\left(\theta_{0}\right)=1.6558$ remains below the robust optimal one. It is interesting to look also at the effect that the identification experiment induces in each module. To this aim, we define a local cost $\tilde{J}_{i}$ and a local actual cost $J_{i}$, for a generic module $\mathscr{N}_{i}$ as:

$$
\begin{array}{r}
\tilde{J}_{i}=\sum_{m=1}^{n_{\omega}} \frac{A_{o p t, m}^{2}}{2}\left(\alpha_{y_{r, i}}^{U_{\text {init }}}\left(\omega_{m}\right)+\eta_{u} \alpha_{u_{r, i}}^{U_{i n i t}}\left(\omega_{m}\right)\right) \\
J_{i}=\mathscr{P}_{y_{r, i}}\left(\Phi_{r, o p t}, \theta_{0}\right)+\eta_{u} \mathscr{P}_{u_{r, i}}\left(\Phi_{r, o p t}, \theta_{0}\right)
\end{array}
$$

where $\mathscr{P}_{y_{r, i}}\left(\Phi_{r, o p t}, \theta_{0}\right)\left(\operatorname{resp} \mathscr{P}_{u_{r, i}}\left(\Phi_{r, o p t}, \theta_{0}\right)\right.$ ) is the power of the signal $y_{r, i}(t)$ (resp $\left.u_{r, i}(t)\right)$, computed with $\Phi_{r, o p t}$ and $\theta_{0}$.

In Table I we observe that the local costs $J_{i}$ remain below the robust ones $\tilde{J}_{i}$, due to the fact that the uncertainties associated to the modules $\mathscr{N}_{k}$ play really a little role in the determination of the cost, as we have previously assumed. Note that the costs for $\mathscr{N}_{1}$ are equal to 0 , since in our setting this module does not receive any information.

TABLE I

ACTUAL $J_{i}$ AND ROBUSTIFIED $\tilde{J_{i}}$ LOCAL COSTS

\begin{tabular}{|c||c|c|c|c|c|c|}
\hline & $\mathscr{N}_{1}$ & $\mathscr{N}_{2}$ & $\mathscr{N}_{3}$ & $\mathscr{N}_{4}$ & $\mathscr{N}_{5}$ & $\mathscr{N}_{6}$ \\
\hline$\tilde{J}_{i}$ & 0 & $6.310^{-7}$ & $2.310^{-4}$ & $2.310^{-4}$ & 1.8296 & $9.310^{-4}$ \\
\hline$J_{i}$ & 0 & $1.310^{-7}$ & $410^{-5}$ & $410^{-5}$ & 1.6556 & $1.610^{-4}$ \\
\hline
\end{tabular}

Comparison between Stealth and Non Stealth Configuration. We solve Problem 3 using the LMI solver provided in the Robust Control toolbox in Matlab, but without modifying the module $\mathscr{N}_{5}$, thus in a Non Stealth Configuration as in Fig. 1.

In order to verify the effectiveness of the Stealth Configuration, we check whether the robust costs are lower using the Stealth Configuration. To this aim, we have defined the cost reduction in Stealth Configuration with respect to the Non Stealth one as:

$$
C R=\frac{\tilde{J}^{N S}-\tilde{J}^{S}}{\tilde{J}^{N S}} \times 100
$$

where $\tilde{J}^{N S}$ and $\tilde{J}^{S}$ are the robustified costs obtained in the Non Stealth and Stealth configuration respectively. We obtain a cost reduction $C R=1.2 \%$ for the global cost, while $C R$ for the local costs are listed in Table II. It is interesting that the Stealth Configuration has a little effect on the cost of the to-be-identified module, but its effectiveness is important when looking at the other modules, thus proving to be an important tool for attenuating the effects of the excitation signal on the rest of the network. Again, $C R$ for $\mathscr{N}_{1}$ is equal to 0 , since in our setting this module does not receive any information.

TABLE II

COST REDUCTION $C R$ OBTAINED FOR EACH LOCAL COST $\tilde{J_{i}}$.

\begin{tabular}{|c||c|c|c|c|c|c|}
\hline Module & $\mathscr{N}_{1}$ & $\mathscr{N}_{2}$ & $\mathscr{N}_{3}$ & $\mathscr{N}_{4}$ & $\mathscr{N}_{5}$ & $\mathscr{N}_{6}$ \\
\hline$C R[\%]$ & 0 & 93.5 & 93.9 & 93.9 & 0.02 & 93.9 \\
\hline
\end{tabular}

\section{CONCLUSIONS}

This paper represents a first contribution towards the extension of the least costly experiment design paradigm [6] to a networked case. Moreover, the Stealth Configuration has been here extended to consider also the uncertainty of the initial model and used here as tool for reducing the propagation of the excitation signal through the network, providing really promising results when using an uncertainty region that is small enough. Finally, the result in Proposition 1 constitutes a tool to exactly robustify the optimal experiment design with respect to its cost if we consider the single closed-loop case. 


\section{REFERENCES}

[1] J. A. Fax and R. M. Murray, "Information flow and cooperative control of vehicle formations," IEEE transactions on automatic control, vol. 49, no. 9, pp. 1465-1476, 2004.

[2] R. Olfati-Saber, J. A. Fax, and R. M. Murray, "Consensus and cooperation in networked multi-agent systems," Proceedings of the IEEE, vol. 95, no. 1 , pp. 215-233, 2007

[3] P. Seiler, A. Pant, and K. Hedrick, "Disturbance propagation in vehicle strings," IEEE Transactions on automatic control, vol. 49, no. 10, pp. 1835-1842, 2004.

[4] M. Andreetto, M. Pacher, D. Fontanelli, and D. Macii, “A cooperative monitoring technique using visually servoed drones," in Environmental, Energy and Structural Monitoring Systems (EESMS), 2015 IEEE Workshop on. IEEE, 2015, pp. 244-249.

[5] L. Ljung, System Identification-Theory for the User 2nd edition PTR Prentice-Hall. Upper Saddle River, USA, 1999.

[6] X. Bombois, G. Scorletti, M. Gevers, P. M. Van den Hof, and R. Hildebrand, "Least costly identification experiment for control," Automatica, vol. 42, no. 10 , pp. 1651-1662, 2006.

[7] P. M. Van den Hof, A. Dankers, P. S. Heuberger, and X. Bombois, "Identification of dynamic models in complex networks with prediction error methods-basic methods for consistent module estimates," Automatica, vol. 49, no. 10, pp. 2994-3006, 2013.

[8] A. Dankers, P. M. Van den Hof, X. Bombois, and P. S. Heuberger, "Identification of dynamic models in complex networks with prediction error methods: Predictor input selection," IEEE Transactions on Automatic Control, vol. 61, no. 4, pp. 937-952, 2016.

[9] X. Bombois, A. Korniienko, H. Hjalmarsson, and G. Scorletti, "Optimal identification experiment design for the interconnection of locally controlled systems," Automatica, vol. 89, pp. 169-179, 2018.

[10] M. Potters, X. Bombois, M. Forgione, P. Modén, M. Lundh, H. Hjalmarsson, and P. Van den Hof, "Optimal experiment design in closed loop with unknown, nonlinear and implicit controllers using stealth identification," in Control Conference (ECC), 2014 European. IEEE, 2014 , pp. 726-731.

[11] H. Hjalmarsson, "System identification of complex and structured systems," in Control Conference (ECC), 2009 European. IEEE, 2009, pp. 3424-3452.

[12] A. Kumar and S. Narasimhan, "Robust plant friendly optimal input design," IFAC Proceedings Volumes, vol. 46, no. 32, pp. 553-558, 2013.

[13] M. Forgione, X. Bombois, and P. M. Van den Hof, "Data-driven model improvement for model-based control," Automatica, vol. 52, pp. 118-124, 2015.

[14] C. R. Rojas, J. S. Welsh, G. C. Goodwin, and A. Feuer, "Robust optimal experiment design for system identification," Automatica, vol. 43, no. 6, pp. $993-1008,2007$.

[15] X. Bombois, M. Gevers, G. Scorletti, and B. D. Anderson, "Robustness analysis tools for an uncertainty set obtained by prediction error identification," Automatica, vol. 37, no. 10, pp. 1629-1636, 2001

[16] M. G. Safonov, Stability and robustness of multivariable feedback systems. MIT press, 1980.

[17] S. Boyd and L. Vandenberghe, Convex optimization. Cambridge university press, 2004.

[18] I. Landau, D. Rey, A. Karimi, A. Voda, and A. Franco, “A flexible transmission system as a benchmark for robust digital control," European Journal of Control, vol. 1, no. 2, pp. 77-96, 1995. 